\title{
Variations of Bergman Kernels for Some Explicitly Given Families of Planar Domains
}

\author{
Y.Y. Wang \\ Department of Mathematics \\ Tongji University \\ Shanghai, China
}

\begin{abstract}
We study the parameter dependence of the Bergman kernels on some planar domains depending on complex parameter $\zeta$ in nontrivial "pseudoconvex" ways. Smoothly bounded cases are studied at first: It turns out that, in an example where the domains are discs, the Levi form for the logarithm of the Bergman kernels with respect to $\zeta$ approaches to $\infty$ as the point tends to the complement of a point in the boundary. Further, in contrast to this, in the cases where the boundaries of the domains are nonsmooth, such as discs with slits interesting phenomena isobserved.
\end{abstract}

Keywords-bergman kernel; plurisubharmonic function; pseudoconvex domain

\section{INTRODUCTION AND RESULTS}

Bergman kernel and Bergman metric have been studied in detail in the case of bounded strongly pseudoconvex domains with $C^{\infty}$ boundary. For such domains C. Fefferman [4] (1974) found a remarkable asymptotic formula for the Bergman kernel form. He used it to show that suitable geodesics of the Bergman metric approach the boundary of the domain in a "pseudotransverse" manner, and therefore that biholomorphicmappings of strongly pseudoconvex domains extend smoothly to the boundaries. In 1978, using Fefferman's asymptotic formula, P.Klembeck[7] showed, for a bounded strongly pseudoconvex domain $\Omega$ with $C^{\infty}$ smooth boundary, that the holomorphic sectional curvature of Bergman pseudometric near the boundary of $\Omega$ approaches to the negative constant $-2 /(n+1)$ which is the holomorphic sectional curvature of the Bergman metric of the unit ball in $C^{n}$. For some other results on the boundary behavior of the Bergman kernel, refer to [3], [5], [6], [9].

In 2004, F. Maitani and H. Yamaguchi [8] brought a new viewpoint by studying the variation of the Bergman metrics on the Riemann surfaces. Let us briefly recall it.

Let $B$ be a disk in the complex $\zeta$-plane, $\mathrm{D}$ be a domain in the products pace $B \times \mathbb{C}_{z}$, and let $\pi$ be the first projection from $B \times \mathbb{C}_{z}$ to $B$ which is proper and smooth and $D_{\zeta}=\pi^{-1}(\zeta)$ be a domain in $\mathbb{C}_{z}$. Put

$$
\partial \mathrm{D}=\bigcup_{\zeta \in B}\left(\zeta, \partial D_{\zeta}\right)
$$

Let $\zeta \in B \quad, \quad z \in D_{\zeta}$ and consider the potential $\psi(\zeta, t, z)$ for $\left(D_{\zeta}, z\right)$ which is a complex valued harmonic function on $D_{\zeta} \backslash\{z\}$ vanishing on the boundary of $D_{\zeta}$, and decompose $d_{t} \psi(\zeta, t, z)$ into

$$
d_{t} \psi(\zeta, t, z)=\mathrm{L}(\zeta, t, z) d t+\overline{\mathrm{K}(\zeta, t, z)} \overline{d t}
$$

On $D_{\zeta} \backslash\{z\}$, where

$$
\mathrm{L}(\zeta, t, z)=\frac{\partial \psi(\zeta, t, z)}{\partial t}, \mathrm{~K}(\zeta, t, z)=\frac{\partial \overline{\psi(\zeta, t, z)}}{\partial t} .
$$

Let $g(\zeta, t, z)$ be the Green function and $\lambda(\zeta, z)$ the Robin constant for $\left(D_{\zeta}, z\right)$, then,

$$
g(\zeta, t, z)=\log \frac{1}{|t-z|}+\lambda(\zeta, z)+h(\zeta, t, z) .
$$

Here, $h(\zeta, t, z)$ is harmonic for $t$ in a neighborhood of $z$ in $D_{\zeta}$ such that $h(\zeta, z, z)=0 \quad$ for $\zeta \in B$.

Let $\varphi(\zeta, t)$ be a defining function of $\partial \mathrm{D}$ in $B \times \mathbb{C}_{t}$ and define

$k_{2}(\zeta, t)=\left(\frac{\partial^{2} \varphi}{\partial \zeta \partial \bar{\zeta}}\left|\frac{\partial \varphi}{\partial t}\right|^{2}-2 \Re\left\{\frac{\partial^{2} \varphi}{\partial \zeta \partial \bar{t}} \frac{\partial \varphi}{\partial \zeta} \frac{\partial \varphi}{\partial \bar{t}}\right\}+\left|\frac{\partial \varphi}{\partial \zeta}\right|^{2} \frac{\partial^{2} \varphi}{\partial t \partial \bar{t}}\right) /\left|\frac{\partial \varphi}{\partial t}\right|^{3}$.

Then, the following variation formulae for the Bergman kernels $K_{D_{\zeta}}(z, z)$ on the diagonal can be obtained.

Theorem 0.1[8] It holds for $(\zeta, z) \in \mathrm{D}$

$$
\begin{gathered}
\frac{\partial^{2} K_{D_{\zeta}}(z, z)}{\partial \zeta \partial \bar{\zeta}}=\frac{1}{4} \int_{\partial D_{\zeta}} k_{2}(\zeta, t)\left(|\mathrm{L}(\zeta, t, z)|^{2}+|\mathrm{K}(\zeta, t, z)|^{2}\right) d s_{t} \\
+\iint_{D_{\zeta}}\left(\left|\frac{\partial \mathrm{L}(\zeta, t, z)}{\partial \bar{\zeta}}\right|^{2}+\left|\frac{\partial \mathrm{K}(\zeta, t, z)}{\partial \bar{\zeta}}\right|^{2}\right) d x d y .
\end{gathered}
$$

Using Theorem 0.1 , the following two properties of variation of the Bergman kernels on the diagonal can be obtained.

Theorem 0.2 [8] Let $\mathrm{D}$ be a pseudoconvex domain over $B \times \mathbb{C}_{z}$ with smooth boundary, then $\log K_{D_{\zeta}}(z, z)$ is plurisubharmonic on D. 
Theorem 0.3[8] Let $\mathrm{D}$ be a pseudoconvex domain over $B \times \mathbb{C}_{z}$ with smooth boundary. If, for each $\zeta \in B, \partial \mathrm{D}$ has at least one strictly pseudoconvex point, then $\log K_{D_{\zeta}}(z, z)$ is a strictly plurisubharmonicfunction on $\mathrm{D}$.

In 2006, B. Berndtsson generalized the Theorem 0.2 to higher dimension and proved that,

Theorem 0.4[2] Let $\mathrm{D}$ be a pseudoconvex domain in $C_{\zeta}^{k} \times C_{z}^{n}$ and $\phi$ be a plurisubharmonic function on D. For each $\zeta$ let $D_{\zeta}$ denote the $n$-dimensional slice $D_{\zeta}:=\left\{z \in C^{n} \mid(\zeta, z) \in D\right\}$ and by $\phi^{\zeta}$ the restriction of $\phi$ to $D_{\zeta}$. Let $K_{D_{\zeta}}(z, z)$ be the Bergman kernels of Bergman space $A_{\zeta}^{2}\left(D_{\zeta}, e^{-\phi^{\zeta}}\right)$. Then, the function $\log K_{D_{\zeta}}(z, z)$ is plurisubharmonicor identically equal to $-\infty$ on $\mathrm{D}$.

These Theorems give us little information about the boundary behavior of the Bergman kernels on the diagonal, but, for any invariant metric on a domain $\Omega \subset C^{n}$, an important characteristic is its boundary behavior. We now study the boundary behavior of the Levi form for the logarithm of Bergman kernels with respect to the parameter $\zeta$ by using complete explicit formulae of Bergman kernels on certain families. This enables us to see precisely how the Bergman kernels depend on the parameter $\zeta$.

Firstly, we consider parameter domains with smooth boundaries which are family of discs. The considered family of discs is

$$
\begin{gathered}
\mathrm{C}=\bigcup_{\zeta \in B}\{\zeta\} \times C_{\zeta} \text { where } B=\mathrm{D}_{\zeta}, \\
C_{\zeta}=\left\{z \in C_{z} \| z-e^{i \theta(\zeta)}<1\right\} .
\end{gathered}
$$

Here, $\theta(\zeta)$ is a real-valued analytic function and $\theta(0)=0$.

We have the following result on this pseudoconvex domain.

Theorem 1.The Levi form of $\log K_{C_{\zeta}}(z, z)$ with respect to $\zeta$ approaches to $\infty$ when $(\zeta, z) \in \mathrm{C}$ tends to $\partial \mathrm{C} \backslash\{(0,0)\}$ but depends on tan $\arg z$ when $(\zeta, z) \in \mathrm{C}$ tends to $(0,0)$ in a nontrivial way. When $(\zeta, z)$ tends to $(0,0)$ in a nontrivial way, if $\tan \arg z$ tends to $\infty$ then $\partial^{2} \log K_{C_{\zeta}}(z, z) / \partial \zeta \partial \bar{\zeta}$ approaches to $\infty$, otherwise, $\partial^{2} \log K_{C_{\zeta}}(z, z) / \partial \zeta \partial \bar{\zeta}$ approaches to a positive nonzero constant which depends on $\tan \arg z$.

Here, the point $(\zeta, z)$ tends to the boundary in a nontrivial way means that $\zeta$ tends to a fixed point firstly, then $z$ tends to the boundary of the base domain $A_{\zeta}$. By parity of reasoning, we will repeat no more later.

Secondly, we investigate the boundary behavior of the Bergman kernels on particular pseudoconvex domain with non-smooth boundary. Since the variation formulae in Theorem 0.1 does not make sense in the boundary when $\partial \mathrm{D}$ is not smooth, it is natural to ask what will happen to the Bergman kernels on the diagonal in this case. We shall give an answer to this question in a family of discs with slits.

The considered family of discs with slitsis

$$
D_{\zeta}=\left\{z \in \mathrm{D}_{z} \mid z \neq s \zeta, s \geq 1\right\},
$$

where $\zeta \in B$ with $B=\{\zeta \in \mathbb{C} \| \zeta-1|<\delta,| \zeta \mid<1\}$ and define

$$
\mathrm{D}=\bigcup_{\zeta \in B}\{\zeta\} \times D_{\zeta} .
$$

Then the following result holds.

Theorem 2. The Levi form of $\log K_{D_{\zeta}}(z, z)$ with respect to $\zeta$ approaches to $\infty$ when $(\zeta, z) \in \mathrm{D}$ tends to $(1,1) \in \partial \mathrm{D}$ in a nontrivial way and approaches to 0 when $(\zeta, z)$ tends to $(1, \pm i) \in \partial \mathrm{D}$ in a nontrivial way. Otherwise, $\partial^{2} \log K_{C_{\zeta}}(z, z) / \partial \zeta \partial \bar{\zeta}$ approaches to a positive non-zero constant.

\section{PRELIMINARIES}

We briefly present here certain results underlying the proofs of Theorems. This exposition is adapted to our special cases.

The Bergman kernel of a domain $\Omega \subset \mathbb{C}^{n}$ is a reproducing kernel for the Hilbert space of all square integrable holomorphicfunctions on $\Omega$. In what follows, let $\Omega$ be a boundeddomain in $\mathbb{C}^{n}$, let $A^{2}(\Omega)$ be the space of square integrable holomorphic functions on $\Omega$.And let $\left\{\phi_{j}(z)\right\}_{j=0}^{\infty}$ be a complete orthonormal basis for $A^{2}(\Omega)$. Then the Bergman kernel $K_{\Omega}(z, w)$ is identified with the following series:

$$
K_{\Omega}(z, w)=\sum_{j=0}^{\infty} \phi_{j}(z) \overline{\phi_{j}(w)},
$$

which is independent of the choice of orthonormal basis. For $z=w$,one has

$$
K_{\Omega}(z, z)>0 \text {. }
$$

The Bergman kernel satisfies the following transformation formula.

Proposition 2.1.Let $f: \Omega^{\mathrm{TM}} \quad D$ be abiholomorphic 
mapping between $\Omega$ and $D$. Then,

$K_{\Omega}(z, w)=K_{D}(f(z), f(w)) \operatorname{det} f^{\prime}(z) \overline{\operatorname{det} f^{\prime}(w)}$.

By Cauchy's estimate it is easy to see that $K_{\Omega}(z, w)$ is a $C^{\infty}$ function on $\Omega \times \Omega$ and on the diagonal, it can bere presented as

$K_{\Omega}(z, z)=\sup \left\{|f(z)|^{2} \mid f \in A^{2}(\Omega),\|f(z)\|_{A^{2}(\Omega)}=1\right\}$ for $\forall z \in \Omega$.

\section{PROOFS OF THEOREMS}

We next accomplish the proofs of Theorems that are given in the introduction.

\section{A. Case of the Family of Discs}

\section{Proof the theorem 1.}

Using the Proposition 2.1, we can get the Bergman kernels

$$
K_{C_{\zeta}}(z, z)=\frac{1}{\pi\left(1-\left|z+e^{i \theta(\zeta)}\right|^{2}\right)^{2}} .
$$

So,

$$
\frac{\partial^{2} \log K_{C_{\zeta}}(z, z)}{\partial \zeta \partial \bar{\zeta}}=4\left|\theta_{\zeta}\right|^{2} \frac{|z|^{2}\left(\Re\left(z e^{-i \theta(\zeta)}\right)+2\right)}{\left(|z|^{2}+2 \Re\left(z e^{-i \theta(\zeta)}\right)\right)^{2}}
$$

Moreover, the condition $\theta(0)=0$ induces that

$$
\lim _{\zeta \rightarrow 0} \frac{\partial^{2} \log K_{C_{\zeta}}(z, z)}{\partial \zeta \partial \bar{\zeta}}=4\left|\theta_{\zeta}\right|^{2} \frac{|z|^{2}(\operatorname{Re} z+2)}{\left(1-|z+1|^{2}\right)^{2}} .
$$

Then, from (1), if $(\zeta, z) \in \mathrm{C} \quad$ tends to $\partial \mathrm{C} \backslash\{(0,0),(0,-2)\}$ then $\partial^{2} \log K_{C_{\zeta}}(z, z) / \partial \zeta \partial \bar{\zeta}$ tends to $\infty$. Also, from (1) we have

$\lim _{z \rightarrow-2} \lim _{\zeta \rightarrow 0} \frac{\partial^{2} \log K_{C_{\zeta}}(z, z)}{\partial \zeta \partial \bar{\zeta}}=\lim _{z \rightarrow-2} \lim _{\zeta \rightarrow 0} 4\left|\theta_{\zeta}\right|^{2} \frac{1}{(2+\operatorname{Re} z)^{2}}=\infty$.

That is, if $(\zeta, z) \in \mathrm{C}$ tends to $(0,-2) \in \partial \mathrm{C}$ then

$\partial^{2} \log K_{C_{\zeta}}(z, z) / \partial \zeta \partial \bar{\zeta}$ tends to $\infty$ with order 1 .

Finally, we consider the boundary point $(0,0)$.

Let $z=x+i y$, then

$$
\lim _{\zeta \rightarrow 0} \frac{\partial^{2} \log K_{C_{\zeta}}(z, z)}{\partial \zeta \partial \bar{\zeta}}=4\left|\theta_{\zeta}\right|^{2} \frac{(x+2)}{\left(x^{2}+y^{2}+4 x+\frac{4}{\left(1+(y / x)^{2}\right)}\right)} .
$$

Therefore, when $(\zeta, z)$ tends to $(0,0)$, the Levi form of $\log K_{C_{\zeta}}(z, z)$ with respect to $\zeta$ is dependent on $\tan \arg z$ and if $\tan \arg z$ tends to $\infty$ the Levi form of $\log K_{C_{\zeta}}(z, z)$ with respect to $\zeta$ tends to $\infty$, otherwise, the Levi form of $\log K_{C_{\zeta}}(z, z)$ with respect to $\zeta$ tends to a positive non-zero constant which depends on $\tan \arg z$.

B. Case of the Family of Discs with Slits

We will now proceed with the proof of Theorem 2.

Proof the theorem 2. Define that

$$
z=E_{\zeta}(w):=e^{-i \theta} K^{-1}\left(e^{-t} K(w)\right),
$$

where $K(w)=w /(1+w)^{2}$ is the Koebe function, $\zeta=e^{-i \theta} K^{-1}\left(e^{-t} / 4\right)$ with $t>0, \theta \in[0,2 \pi)$, this is inspired by [1].

For each $\zeta, E_{\zeta}(w)$ maps the unit disc D in the w plane to $D_{\zeta}$ which is the unit disc in the complex z-plane minus a segment $\left[\zeta, e^{-i \theta}\right)$.

Then the inverse mapping of $E_{\zeta}(w)$ is

$$
w=E_{\zeta}^{-1}(z)=K^{-1}\left(e^{t} K\left(e^{i \theta} z\right)\right) .
$$

We now can get the power series expansion of $E_{\zeta}^{-1}(z)$ with respect to the parameter $\zeta$ in a neighborhood of $\zeta=1$, that is, in a neighborhood of $(t, \theta)=(0,0)$ which is

$$
\begin{gathered}
E_{\zeta}^{-1}(z)=K^{-1}\left(e^{t} K\left(e^{i \theta} z\right)\right) \\
=z+\frac{K(z)}{K^{\prime}(z)} t+i z \theta+\left(\frac{K(z)}{K^{\prime}(z)}-\frac{1}{2} \frac{K(z)^{2} K^{\prime \prime}(z)}{\left(K^{\prime}(z)\right)^{3}}\right) t^{2} \\
-\frac{1}{2} z \theta^{2}+\frac{1}{2}\left(1-\frac{K(z)}{K^{\prime}(z)} \frac{K^{\prime \prime}(z)}{K^{\prime}(z)}\right) i z t \theta+O\left(t^{3}\right)+O\left(\theta^{3}\right) \\
=z+\frac{z(1+z)}{(1-z)} t+i z \theta+\frac{1}{2} \frac{z(1+z)\left(1+2 z-z^{2}\right)}{(1-z)^{3}} t^{2} \\
-\frac{1}{2} z \theta^{2}+\frac{1}{2} \frac{1+2 z-z^{2}}{(1-z)^{2}} i z t \theta+O\left(t^{3}\right)+O\left(\theta^{3}\right) .
\end{gathered}
$$

On the other hand, as it is well known, the Bergman kernel of the unit disc D on the diagonal is

$$
K_{\mathrm{D}}(w, w)=\frac{1}{\pi} \frac{1}{\left(1-|w|^{2}\right)^{2}} .
$$

Then, by Proposition 2.1, the Bergman kernels of $D_{\zeta}$ on the diagonal are given by

$$
K_{D_{\zeta}}(z, z)=\frac{1}{\pi} \frac{1}{\left(1-\left|E_{\zeta}^{-1}(z)\right|^{2}\right)^{2}}\left|\left(E_{\zeta}^{-1}(z)\right)_{z}\right|^{2} .
$$

Furthermore, we can calculate that 


$$
\begin{gathered}
\lim _{\zeta \rightarrow 1} \frac{\partial^{2} \log \left(1-\left|E_{\zeta}^{-1}(z)\right|^{2}\right)}{\partial \zeta \partial \bar{\zeta}}=\frac{-2|z|^{2} \operatorname{Re}((1+z) /(1-z))}{\left(1-|z|^{2}\right)}, \\
\lim _{\zeta \rightarrow 1} \frac{\partial^{2} \log \left|\left(E_{\zeta}^{-1}(z)\right)_{z}\right|^{2}}{\partial \zeta \partial \bar{\zeta}}=\frac{1}{4} \operatorname{Re}\left(\frac{1-3 z^{2}}{(1-z)^{2}}\right) .
\end{gathered}
$$

Now let $z=r e^{i \theta} \in D_{\zeta}$, we conclude that

$$
\lim _{\zeta \rightarrow 1} \frac{\partial^{2} \log K_{D_{\zeta}}(z, z)}{\partial \zeta \partial \bar{\zeta}}=\frac{1}{4} \frac{1+r^{2} \cos 2 \theta}{1+r^{2}-2 r \cos \theta} .
$$

Then

$$
\lim _{r \rightarrow 1} \lim _{\zeta \rightarrow 1} \frac{\partial^{2} \log K_{D_{\zeta}}(z, z)}{\partial \zeta \partial \zeta}=\frac{1}{4}\left((1-\cos \theta)+\frac{1}{(1-\cos \theta)}-2\right)
$$

If $\zeta \rightarrow 1$ then the singular point of the boundary $D_{\zeta}$ tends to $z=1$. We see from (2) that

(1) $\partial^{2} \log K_{D_{\zeta}}(z, z) / \partial \zeta \partial \bar{\zeta}$ tends to $\infty$ with order 2 as $(\zeta, z) \in \mathrm{D}$ tends to $(1,1)$.

(2) $\partial^{2} \log K_{D_{\zeta}}(z, z) / \partial \zeta \partial \bar{\zeta}$ tends to 0 with order 2 as $(\zeta, z) \in \mathrm{D}$ tends to $(1, \pm i)$.

(3) $\partial^{2} \log K_{D_{\zeta}}(z, z) / \partial \zeta \partial \bar{\zeta}$ tends to a positive number as $(\zeta, z) \in \mathrm{D}$ tends to $(1, z) \in \partial \mathrm{D}$ here $z \neq 1, \pm i$.

\section{ACKNOWLEDGEMENTS}

Project supported by NSFC No. 11031008 and NSFS No. 13ZR1444100.

\section{REFERENCES}

[1] L. V. Ahlfors, Conformal Invariants, McGraw-Hill, 1973.

[2] B. Berndtsson, Subharmonicity properties of the Bergman kernel and some otherfunctions associated to pseudoconvex domains, AnnalesDeL'Institute Fourier (Grenoble)56, pp. 1633-1662,2006.

[3] K. Diederich, and G. Herbort, Geometric and analytic boundary invariants on pseudoconvex domains. Comparison results, Journal of Geometric Analysis3, pp. 237-267, 1993.

[4] C. Fefferman, The Bergman kernel and biholomorphic mappings of pseudoconvexdomains, InventionsMathematicae26,pp. 1-65, 1974.

[5] G. Herbort, The growth of the Bergman kernel onpseudoconvex domains of homogeneous finite diagonal type,Nagoya Mathematical Journal, 126, 1-24, 1992.

[6] K. Diederich and T. Ohsawa, On the parameter dependece of solutions to the equation, MathematischeAnnalen, 289, 581-588, 1991.

[7] P. Klembeck, Kahler metrics of negative curvature, the Bergman metric near theboundary and the Kobayashi metric on smooth bounded pseudoconvex sets, IndianUniversity Mathematics Journal27, 275-282, 1978.

[8] F. Maitani and H. Yamaguchi, Variation of Bergman metrics on Riemann surfaces, MathematischeAnnalen, 330, 477-489, 2004.

[9] J.D.McNeal,LowerboundsontheBergmanmetricnearapointoffinit etype, Annalsof Mathematics, 136, 339-360, 1992.(1992) 